\title{
Software Polarization Spectrometer "PolariS"
}

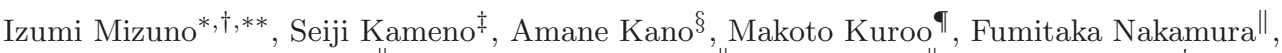 \\ Noriyuki Kawaguchi", Katsunori M. Shibata", Seisuke Kujil and Nario Kuno ${ }^{\dagger}$ \\ * Department of Physics and Astronomy \\ Graduate School of Science and Engineering \\ Kagoshima University, 1-21-35 Korimoto \\ Kagoshima 890-0065, Japan \\ ${ }^{\dagger}$ Nobeyama Radio Observatory \\ Minamimaki, Minamisaku \\ Nagano 384-1805, Japan \\ ${ }^{\ddagger}$ Joint ALMA Observatory \\ Alonso de Córdova 3107 Vitacura \\ Santiago 7630355, Chile \\ $\S$ Department of Physics, Faculty of Science \\ Kagoshima University, 1-21-35 Korimoto \\ Kagoshima 890-0065, Japan \\ ฯ Shoyo High School, Tatemachi \\ Hachioji, Tokyo 293-0944, Japan \\ " National Astronomical Observatory of Japan \\ 2-21-1 Osawa, Mitaka, Tokyo 181-8588, Japan \\ **k7330312@kadai.jp
}

Received 2014 April 30; Revised 2014 October 7; Accepted 2014 December 2; Published 2014 December 23

\begin{abstract}
We have developed a software-based polarization spectrometer, PolariS, to acquire full-Stokes spectra with a very high spectral resolution of $61 \mathrm{~Hz}$. The primary aim of PolariS is to measure the magnetic fields in dense star-forming cores by detecting the Zeeman splitting of molecular emission lines. The spectrometer consists of a commercially available digital sampler and a Linux computer. The computer is equipped with a graphics processing unit (GPU) to process FFT and cross-correlation using the Compute Unified Device Architecture (CUDA) library developed by NVIDIA. Thanks to a high degree of precision in quantization of the analog-to-digital converter and arithmetic in the GPU, PolariS offers excellent performances in linearity, dynamic range, sensitivity, bandpass flatness and stability. The software has been released under the MIT License and is available to the public. In this paper, we report the design of PolariS and its performance verified through engineering tests and commissioning observations.
\end{abstract}

Keywords: Polarimetry, radio spectroscopy, GPUs, software.

\section{Introduction}

\subsection{Polarization spectrometer}

A polarization spectrometer is a device to measure the polarimetric properties of incident radiation as a function of frequency. The Stokes parameters, $I$, $Q, U$, and $V$, are a representation of polarized light, and are all measurements of flux density. The total flux density is denoted by $I$, and the level of general elliptical polarization is described by nonzero values of $Q, U$, and $V$.

A typical astronomical receiving system is sensitive to either linear or circular polarization. In both cases, the receiving system will measure two products, either two orthogonal linear polarizations or two opposing circular polarizations. In order to obtain all four Stokes parameters from the two observables, both auto- and cross-correlation 
products must be formed from the received polarizations.

In a modern radio telescope, received signals are numerically processed to output correlation products in a digital polarization spectrometer. The XPOL correlation polarimeter system is installed on the IRAM 30-m telescope (Thum et al., 2008) and the VESPA digital polarization spectrometer of the system yields a $40-\mathrm{kHz}$ spectral resolution across a $120-\mathrm{MHz}$ bandwidth. The ACS spectrometer is installed on the Green Bank Telescope and offers a $49-\mathrm{kHz}$ spectral resolution in a $12.5-\mathrm{MHz}$ bandwidth. The WAPP correlator (Dowd et al., 2000) is used in the Arecibo radio telescope mainly for pulsar observations, with a $0.195 \mathrm{MHz}$ resolution. Such hardware-based polarization spectrometers are not sufficiently flexible nor scalable for finer spectral resolutions, wider bandwidths, etc.

A software-based polarization spectrometer, composed by a personal computer, is an alternative implementation to bring flexibility and scalability. Recent high-performance computing system with standard off-the-shelf Graphics Processing Units (GPUs) provides solutions for real-time spectroscopy (e.g. Magro et al., 2011; Barsdell et al., 2012) with high-level programming interfaces such as CUDA (NVIDIA Corporation, 2007). The software implementation yields some advantages. It is flexible and scalable when modification of specifications are required. The source codes in programming language are readable, verifiable, and can be shared or updated via a public repository. The processors are off-the-shelf products, which allows us to build copies or to prepare spares at a reasonable cost.

In this paper, we describe the construction of the software-based polarization spectrometer, PolariS. The scientific requirements and specifications to meet are described in Sec. 1.2. The basic design and costs are presented in Sec. 2. The verification is reported in Sec. 3. And in Sec. 4, field test results of observations toward radio sources are demonstrated.

\subsection{Science target}

The primary aim of Polaris is to measure the strength of the magnetic fields in the star-forming cores of molecular clouds through the Zeeman effect of emission lines. The magnetic field is a key control parameter that can mediate gravitational collapse of these cores (Spitzer, 1968), and the Zeeman effect is one of the most reliable probes to measure the strength of magnetic fields. While magnetic field strength in the interstellar medium has been measured toward diffuse and dark clouds with Hi and OH (Troland \& Heiles, 1986; Goodman et al., 1989; Crutcher et al., 1993), star-forming regions with OH masers (Lo et al., 1975; Reid et al., 1980; Gaume \& Mutel, 1987), OH outflows (Norris, 1984; Baart et al., 1986), interstellar $\mathrm{H}_{2} \mathrm{O}$ maser clumps (Fiebig \& Guesten, 1989), and massive star-forming regions with CN radical (Crutcher et al., 1999), those in the star-forming cores have not been measured to date.

According to the relation of gas density and magnetic field strength (Fiebig \& Guesten, 1989), the field strength in the star-forming cores with the gas density of $n_{\mathrm{H}} \sim 10^{4}-10^{5} \mathrm{~cm}^{-3}$ is expected to lie between 100 and $1000 \mu \mathrm{G}$. With typical parameters of the core radius of $\sim 0.1 \mathrm{pc}$ and the mass of $\sim 10 \mathrm{M}_{\odot}$ (Onishi et al., 2002), the gravityequivalent magnetic field strength will be $\sim 100 \mu \mathrm{G}$ (Shu et al., 1987). Thus, measurements of $\sim 100 \mu \mathrm{G}$ magnetic fields in star-forming cores are crucial to discriminate whether the magnetic fields can support a core against gravitational collapse. The thioxoethenylidene (CCS) radical is one of the best molecules to investigate magnetic field strength in star-forming cores because it is abundant in dark cloud cores, its emission lines are intense and narrow, and it shows a relatively large Zeeman split with $0.64 \pm 0.12 \mathrm{~Hz} \mu \mathrm{G}^{-1}$ for the $J_{N}=4_{3}-3_{2}$ transition at $45.379 \mathrm{GHz}$ (Shinnaga \& Yamamoto, 2000).

\subsection{Requirements}

The specification of PolariS must be compliant to measure the Zeeman split of the CCS molecular emission. The expected magnetic field of $\sim 100 \mu \mathrm{G}$ will yield the Zeeman split of $\sim 64 \mathrm{~Hz}$ (Shinnaga \& Yamamoto, 2000) between two orthogonal circular polarizations. The Zeeman split will appear in the Stokes $V$ (circular polarization) spectrum as

$$
V(\nu) \simeq \frac{d I(\nu)}{d \nu} \Delta \nu
$$

where $I(\nu)$ is the Stokes $I$ spectrum as a function of the frequency, $\nu$, and $\Delta \nu$ is the Zeeman split. Our pilot observations of CCS molecules (see Sec. 4) indicate that the line profile toward the Taurus molecular cloud 1 (TMC-1) has $\frac{d I}{d \nu} \sim 0.16 \mathrm{mK} \mathrm{Hz}^{-1}$, which corresponds to $V \sim 10 \mathrm{mK}$ if we assume that $\Delta \nu=64 \mathrm{~Hz}$. 
In our project we use the Z45 receiver (Tokuda et al., 2013; Nakamura et al., 2013), which is capable of receiving two orthogonal linear polarizations. The Stokes $V$ component can be obtained via cross-correlation between two linear polarizations. To measure such a weak and narrow Stokes $V$ feature with Z45, PolariS must specify: (1) measurements of autocorrelation and cross-correlation, (2) the spectral resolution better than the expected Zeeman split of $64 \mathrm{~Hz},(3)$ adequate sensitivity, and (4) frequency stability better than $60 \mathrm{~Hz}$. The total cost was limited to our budget of JPY $2,000,000$.

\section{Design and Development}

Figure 1 shows a schematic diagram of the signal path to a polarization spectrometer. The functions of the digital FX-type polarization spectrometer are (1) to accept the received signal in two orthogonal polarizations in digital, (2) to calculate spectra of the real-time signal streams via FFT, (3) to produce power spectra and cross power spectra by multiplying the spectra, and (4) to integrate the power spectra and output them.

Table 1 lists the specifications of PolariS. The specification of the $61-\mathrm{Hz}$ resolution at 4 or $8 \mathrm{MHz}$ bandwidth requires 65,536 or 131,072 spectral channels. To produce the spectrum in real time, 262,144- or 131,072-point FFT must be accomplished within less than $8 \mathrm{~ms}$. This processing requires 1440 Mflops per IF or 5600 Mflops for all IFs. Adding 262 Mflops for autocorrelation cross products, at least $\sim 6$ Gflops are necessary for realtime processing.

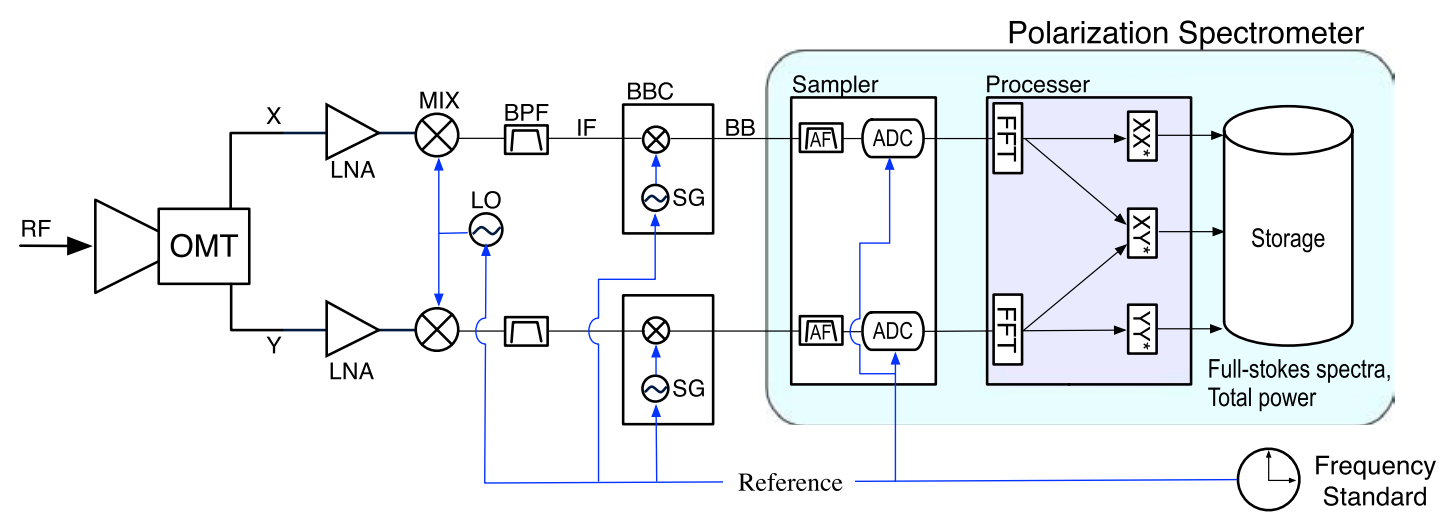

Fig. 1. Schematic diagram of the signal flow of the polarization detection system. The radio frequency (RF) waves incident on the dual polarization receiver will be split into two polarizations ( $X$ and $Y$ ) in the ortho-mode transducer (OMT), amplified in the low noise amplifiers (LNAs), and downconverted in the MIXs (mixers) using the local oscillator (LO) signal referencing the frequency standard. The intermediate frequency (IF) signals through bandpass filters (BPFs) will be downconverted again to basebands (BBs) with baseband converters (BBCs) at desired frequency of signal generator (SGs), and input to a polarization spectrometer. The polarization spectrometer in pale blue consists of a sampler, a processor in pale violet, and storage. The sampler consists of anti-alias filters (AFs) and analog-to-digital converters (ADCs). The digitized signals are converted into spectra though an FFT process, and then multiplications between them are taken to output power and cross spectra $\left(X X^{*}, X Y^{*}\right.$, and $\left.Y Y^{*}\right)$, which yield full Stokes spectra. Detailed signal processing inside the spectrometer is described in Fig. 2.

Table 1. Specifications of PolariS.

\begin{tabular}{|c|c|c|c|}
\hline Feature & \multicolumn{2}{|c|}{ Specification } & Remarks \\
\hline Number of IFs & $4\left(X_{0}, X_{1}, Y_{0}, Y_{1}\right)^{\mathrm{a}}$ & & 2 pols., 2 IFs \\
\hline Bandwidth & $8 \mathrm{MHz} / \mathrm{IF}$ & $4 \mathrm{MHz} / \mathrm{IF}$ & 16 or 8 Msps sampling \\
\hline Quantization & $4 \mathrm{bit} / \mathrm{sample}$ & $8 \mathrm{bit} / \mathrm{sample}$ & \\
\hline Spectral resolution & $131,072 \mathrm{ch} / \mathrm{IF}$ & $65,536 \mathrm{ch} / \mathrm{IF}$ & $61-\mathrm{Hz}$ channel spacing \\
\hline Autocorrelation & 4 real products & & $\left\langle X_{0} X_{0}^{*}\right\rangle,\left\langle X_{1} X_{1}^{*}\right\rangle,\left\langle Y_{0} Y_{0}^{*}\right\rangle,\left\langle Y_{1} Y_{1}^{*}\right\rangle$ \\
\hline Cross-correlation & 2 complex products & & $\left\langle X_{0} Y_{0}^{*}\right\rangle,\left\langle X_{1} Y_{1}^{*}\right\rangle$ \\
\hline Time resolution & $1 \mathrm{~s}$ & & \\
\hline
\end{tabular}

${ }^{\mathrm{a}} X, Y$ stands for two orthogonal polarizations. Suffix indicates basebands. 


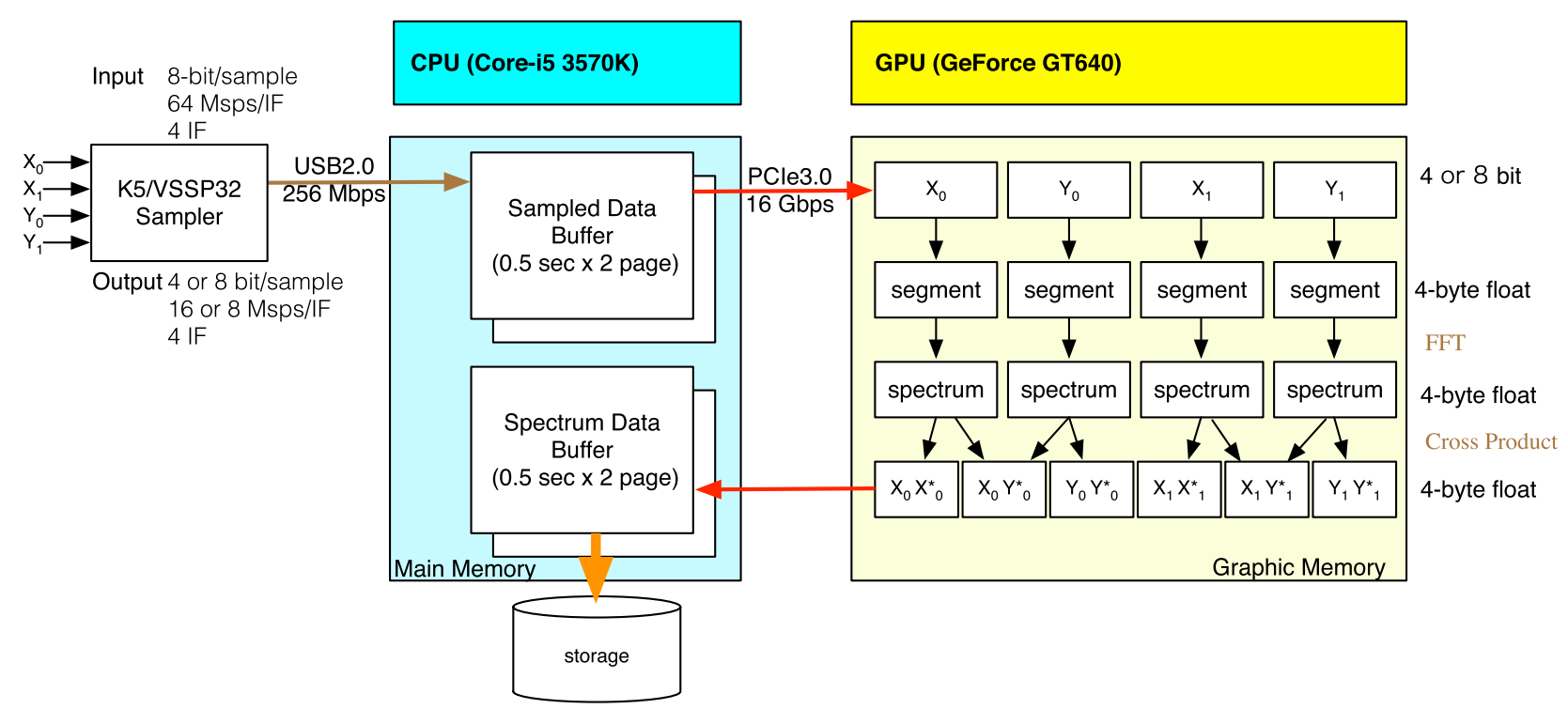

Fig. 2. Schematic diagram of the system and signal flow inside. PolariS consists of the K5/VSSP32 digital sampler and the host computer including CPU in pale blue, GPU in yellow, and storage. The sampler consists of four analog-to-digital converters (ADCs) and accepts four analog IF signals of 32-MHz bandwidth and digitizes them with a sampling speed of 64 Msps/IF and quantization of 8 bit. The digitized signal will be output with 4-bit quantization when we need 8-MHz bandwidth (16 Msps/IF) after applying digital filtering to reduce the total data rate down to $256 \mathrm{Mbps}$ to meet USB2.0. The CPU captures 32 MBytes of data twice a second in the two-page buffer and sends them to GPU memory. The GPU processes data translation (4 or 8-bit integer to 4-byte float), segmentation, FFT, cross products, and accumulation. Each segment contains 131,072 or 262,144 samples to produce 65,536 or 131,072 spectral channels. The (cross) power spectra will be transmitted to the main memory and stored in the file system.

To meet the required specifications within the limited budget, we decided to design PolariS as a software-based spectrometer that consists of a VLBI digitizer, K5/VSSP32 (Kondo et al., 2006b) for data acquisition, and a general-purpose personal computer with a GPU for signal processing. Thanks to recent advances in digital technology, personal computers have become capable of wide-bandwidth signal processing. Furthermore, recent GPUs for video games perform tela floating operations per second (TFLOPS) arithmetic with a low cost.

Figure 2 shows the signal processes in PolariS. The K5/VSSP32 VLBI sampler accepts four analog signals at a maximum bandwidth of $32 \mathrm{MHz}$ per input. After digitization with 8-bit quantization, we employ digital filtering and quantization level reduction (Kondo et al., 2006a) in order to reduce the output data rate to $256 \mathrm{Mbps}$ to accommodate the USB 2.0 interface. The current PolariS system allows two modes: (1) 8-MHz (16 Msps) bandwidth, 4-bit quantization, and (2) 4-MHz bandwidth, 8-bit quantization.

The host computer of PolariS works on the Linux Ubuntu 12.04 LTS operating system. The control and signal-processing software are coded in GNU compiler collection (GCC) and NVIDIA CUDA compiler (NVCC). The cuFFT library is employed to transform time-domain signals into frequency-domain spectra. Total power of each IF signal is measured by histograms of digitized signals (Nakatake et al., 2010). All of the source code is available in the GitHub ${ }^{(a)}$ repository. The computer is equipped with the Intel Core-i5 CPU, 4GB memory, NVIDIA GT640 GPU. It costs JPY 70,000 .

\subsection{Measurement of the Stokes parameters}

PolariS produces power spectra of $\left\langle X X^{*}\right\rangle$ and $\left\langle Y Y^{*}\right\rangle$ and cross power spectra of $\left\langle X Y^{*}\right\rangle$. Full Stokes spectra of $I, Q, U$, and $V$ are derived using these products offline.

If $X$ and $Y$ stand for linear polarizations without any cross talk between them, the Stokes parameters are derived using the equation:

\footnotetext{
${ }^{\mathrm{a}}$ https://github.com/kamenoseiji/PolariS.
} 


$$
\left(\begin{array}{l}
I \\
Q \\
U \\
V
\end{array}\right)=\frac{1}{2}\left(\begin{array}{cccc}
1 & 0 & 0 & 1 \\
\cos 2 \psi_{\mathrm{m}} & -\sin 2 \psi_{\mathrm{m}} & -\sin 2 \psi_{\mathrm{m}} & -\cos 2 \psi_{\mathrm{m}} \\
\sin 2 \psi_{\mathrm{m}} & \cos 2 \psi_{\mathrm{m}} & \cos 2 \psi_{\mathrm{m}} & -\sin 2 \psi_{\mathrm{m}} \\
0 & -i & i & 0
\end{array}\right)\left(\begin{array}{c}
\frac{\left\langle X X^{*}\right\rangle}{G_{X} G_{X}^{*}} \\
\frac{\left\langle X Y^{*}\right\rangle}{G_{X} G_{Y}^{*}} \\
\frac{\left\langle Y X^{*}\right\rangle}{G_{Y} G_{X}^{*}} \\
\frac{\left\langle Y Y^{*}\right\rangle}{G_{Y} G_{Y}^{*}}
\end{array}\right)
$$

where $\psi_{\mathrm{m}}$ is the parallactic angle ( \pm elevation angle for Nasmith optics) and $G_{X}, G_{Y}$ are the voltagedomain complex gain of the receiving system. This conversion is carried out with off-line reduction software.

In a practical receiving system, precise polarization measurements are difficult and require careful instrumental calibration. The measurements are degraded by cross-talk, or leakage, between orthogonal polarizations and fluctuations in the complex gain of the receiving system (Thompson et al., 2001). Detailed calibration schemes will be presented in a forthcoming paper.

\section{Verification}

We have tested the following aspects of performance: spectral resolution (Sec. 3.1), linearity (Sec. 3.2), stability (Sec. 3.3), sensitivity (Sec. 3.4), and cross-correlation capabilities (Sec. 3.5). We used the $\mathrm{Z} 45$ receiver and the $\mathrm{H} 22$ receiver, working at 45 and $22 \mathrm{GHz}$, respectively, installed on the NRO 45-m telescope.

\subsection{Spectral resolution function}

The spectral resolution function is the response of the system to a monochromatic input. It demonstrates the sensitivity of the system as a function of frequency and is an indicator of the coupling between adjacent spectral channels (also known as spectral sidelobes). Any spectral feature measured by the spectrometer is the convolution of the true line shape and this spectral resolution function. The spectral resolution function of PolariS is determined by the FFT process where input data stream is truncated in finite-length segments. PolariS employs a rectangular window function. This makes the spectral response after FFT the sinc function. Thus, the spectral resolution function formed by the cross-multiplication of these FFT products (see Fig. 2) will be a sinc-squared function with the FWHM of 0.886 channel $(54.07 \mathrm{~Hz}$ for
8-MHz bandwidth). A desired apodization window function can be applied offline to reduce the spectral sidelobes. To minimize the information loss through the FFT process, we do not apply apodization in PolariS. We also employed a $50 \%$ overlap of adjacent FFT segments to save sensitivity for very narrow spectral feature by keeping the number of correlation measurements at longer time lags (Thompson et al., 2001).

We confirmed that the measured spectral resolution function coincided with the theoretically expected one through the following tests, whose configuration is shown in Fig. 3. A monochromatic wave was generated by the signal generator (Agilent E8257D) and was injected to PolariS. Its frequency was swept with a $4-\mathrm{Hz}$ step over a $400-\mathrm{Hz}$ range across the reference spectral channel at $68 \mathrm{MHz}$. Since the bandwidth of the monochromatic wave was sufficiently narrow, the response to the reference channel as a function of the frequency separation expressed the spectral resolution function.

To characterize the measured spectral resolution function, we removed the continuum noise level determined by line-free channels. The calibrated power, $P_{\text {ref }}$, as a function of frequency separation of the monochromatic wave is shown in Fig. 4. We applied the least-squares fit with the sinc-squared function as $P_{\text {ref }} \sim a_{0}\left(\frac{\sin \left(\pi\left(a_{1} \nu-a_{2}\right)\right)}{\pi\left(a_{1} \nu-a_{2}\right)}\right)^{2}$, where $a_{0}, a_{1}$, and $a_{2}$ were free parameters that related to amplitude, resolution, and the center frequency, respectively. The best-fit sinc-squared function is displayed in the solid line in Fig. 4. The results corresponded to the spectral resolution of FWHM $=54.02 \pm 0.06 \mathrm{~Hz}$, which was consistent with the ideal expectation.

\subsection{Linearity}

For an ideal spectrometer, the measurement values of spectral power must be proportional to the input power. The linear relation between input and output over a enough power range is crucial for accurate spectroscopy. 


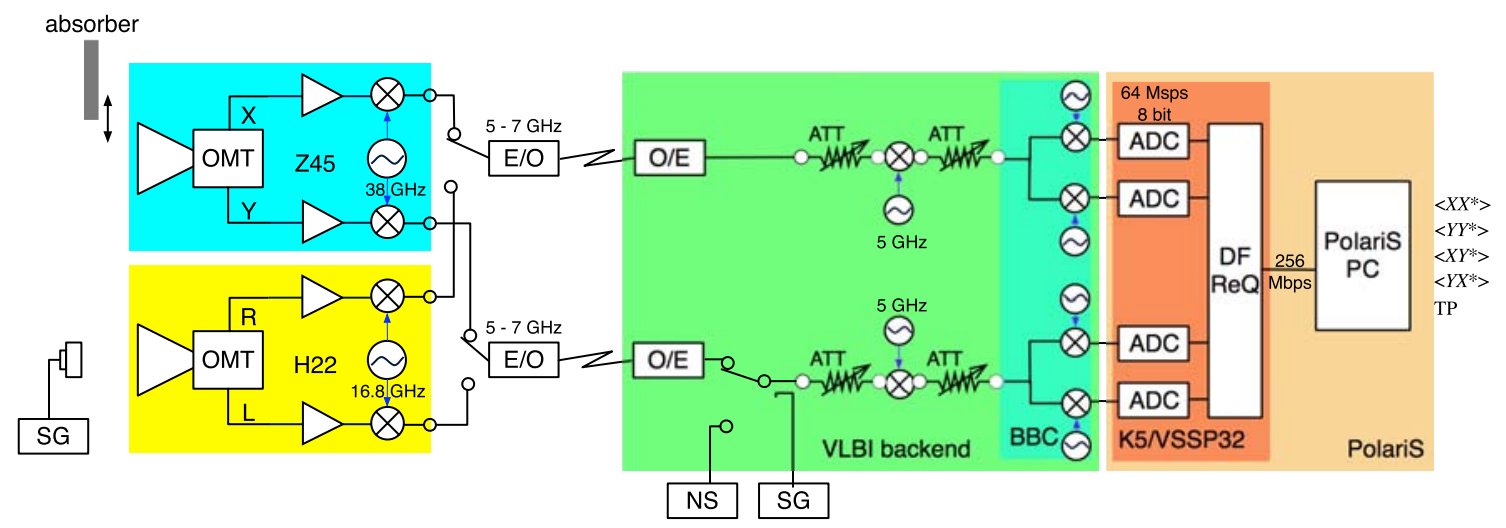

Fig. 3. The test configuration. PolariS in pale orange receives baseband analog signals generated by the BBC of the VLBI backend. Monochromatic wave generated by the SG (Agilent E8257D) or white noise produced by the noise source (NTK 9009ZR) were optionally coupled to measure the spectral resolution function and the linearity in line intensity and total power, respectively. Linear polarizations $(X$ and $Y$ ) and circular polarizations ( $R$ and $L$ ) of the Z45 (pale blue) and H22 (yellow) receivers, respectively, were selectively transmitted to the VLBI backend in green. An absorber was inserted on the feed horn of the Z45 receiver during noise temperature measurements. Linearly polarized monochromatic wave at $22 \mathrm{GHz}$ was injected to the H22 receiver through the feed, generated with a SG (Agilent 83650L) and a transducer with a rectangular waveguide to verify the cross-correlation capability of Polaris.

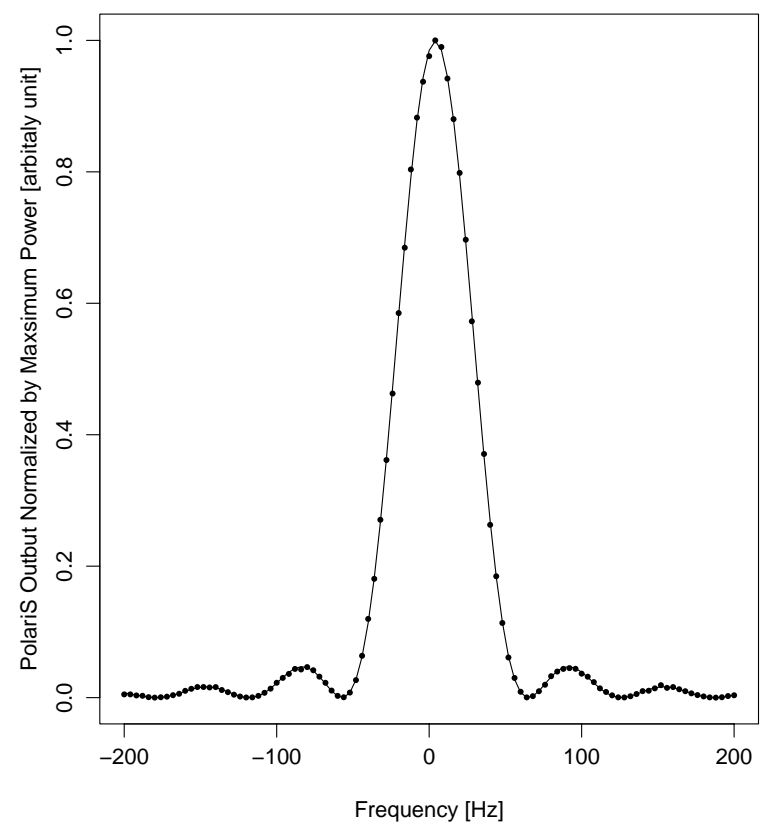

Fig. 4. The spectral resolution of PolariS. Measurements (dots) and the best-fit sinc-squared function with the FWHM of $54.02 \mathrm{~Hz}$ (solid line) are displayed.

We verified the linearity performance in two aspects of measurements: the total power and the spectral line intensity. The former is required to measure system noise temperature and flux densities of continuum sources, while the latter is necessary to measure accurate line intensities and profiles.

\subsubsection{Line intensity}

We used the Z45 receiver pointing to the blank sky and injected a monochromatic wave generated by the signal generator (Agilent E8257D) into the IF signals (see the configuration in Fig. 3) to simulate astronomical spectral-line observations. The frequency of the injected signal was fixed at $5449 \mathrm{MHz}$, corresponding to $1 \mathrm{MHz}$ in the baseband, and the signal power was changed between $-30 \mathrm{dBm}$ and $-75 \mathrm{dBm}$ with a 3 -dB step. To cover a wider power range, we used two additional ATTs of $20 \mathrm{~dB}$ ("high power") and $36 \mathrm{~dB}$ ("low power") for the injection. We set PolariS at the 4-MHz bandwidth, 8-bit quantization and 65,536-channel mode. To measure the line power, we integrated the acquired spectrum for $60 \mathrm{~s}$ at each SG power level and subtracted the continuum baseline as we did for the spectral resolution function (Sec. 3.1). The line power at the reference channel $\left(P_{\text {ref }}\right)$ was compared with the power of injected monochromatic wave, $P_{\text {in }}$.

Figure 5 shows the results, together with the linear regression of $P_{\text {ref }}=b P_{s g}$, where $b$ is the proportional coefficient. The proportional coefficients at "high-power" and "low-power" circumstances were $62.12 \mathrm{~dB}$ and $45.94 \mathrm{~dB}$, respectively. The residuals from the linear regression, $\frac{P_{\text {ref }}-b P_{\text {in }}}{b P_{\text {in }}}$, are also displayed in Fig. 5. The results indicated that the departure from the linear regression was less than $1 \%$ within the power range of $33 \mathrm{~dB}$ and $12 \mathrm{~dB}$ in "high-power" and "low-power" circumstances, respectively. 

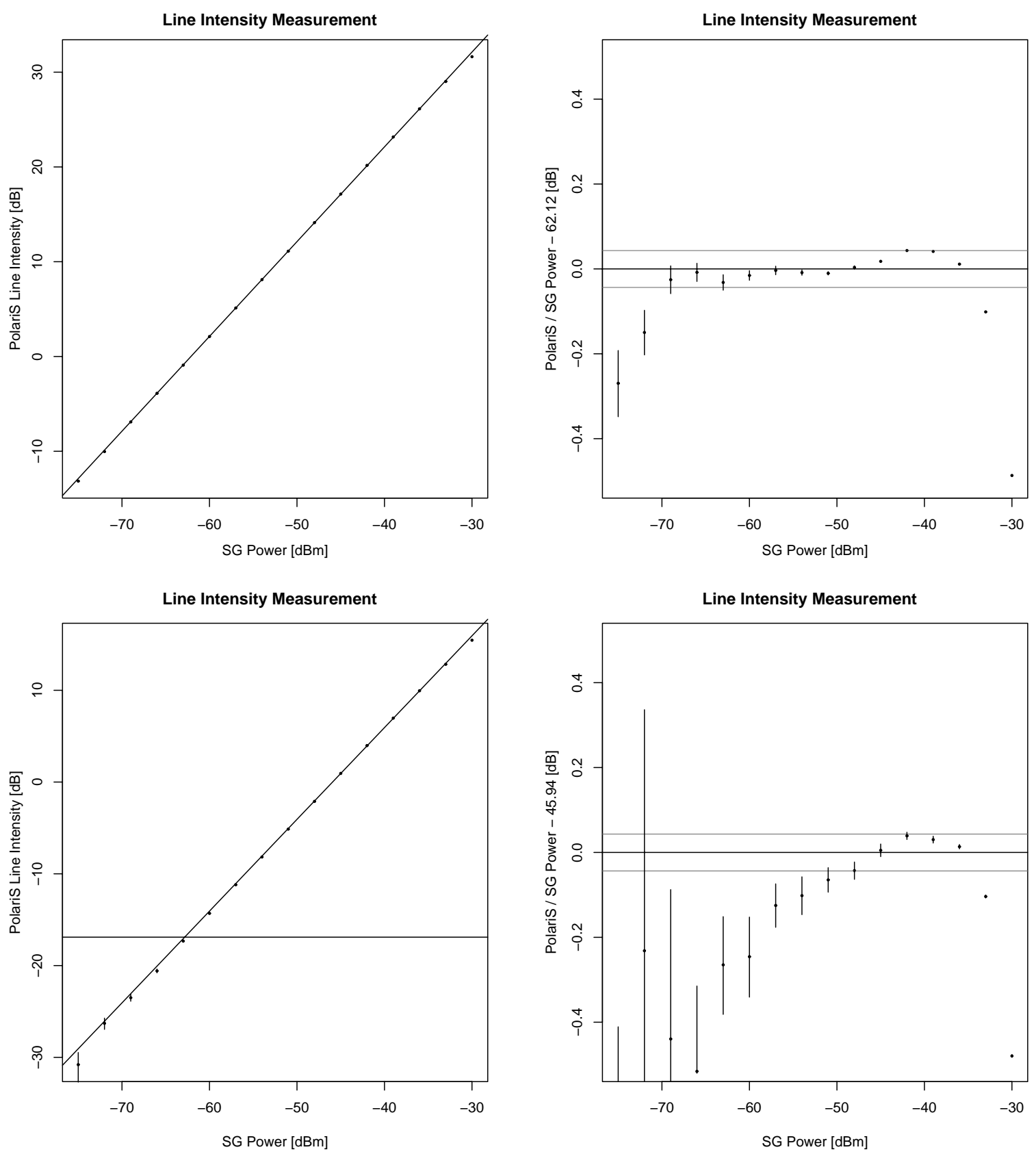

Fig. 5. Linearity in line-intensity measurements. Top and bottom panels stand for "high-power" and "low-power" injection of a monochromatic wave produced in the SG. The low-power injection employs an additional 16-dB attenuator (ATT) compared with the high-power circumstance. (Left): Line intensity measured by PolariS as a function of input power (dots). The solid diagonal line indicates the best-fit linear relation with the slope of unity. The gray horizontal line indicates the standard deviation (SD) of the system noise level. (Right): Departure from the linear relation marked as the solid diagonal line. Horizontal gray lines indicate the departure of $\pm 1 \%$ range.

\subsubsection{Total power}

To verify the linearity of total power measurements, we injected the white noise generated by the noise source (NTK 9009ZR) and adjusted the power level using a combination of two step ATTs covering a $22-\mathrm{dB}$ range by a $1-\mathrm{dB}$ step. We employed the power measurement scheme using the bit distribution of digitized signals in the ADC (Nakatake et al., 2010). The input analog power was monitored using the power meter (HP E4419A) with the power sensor (HP 8481D). The test configuration is shown in Fig. 3, too.

The results are shown in Fig. 6 together with the linear regression of $\log P_{\text {Polaris }} \sim \log P_{\text {in }}+c$, 

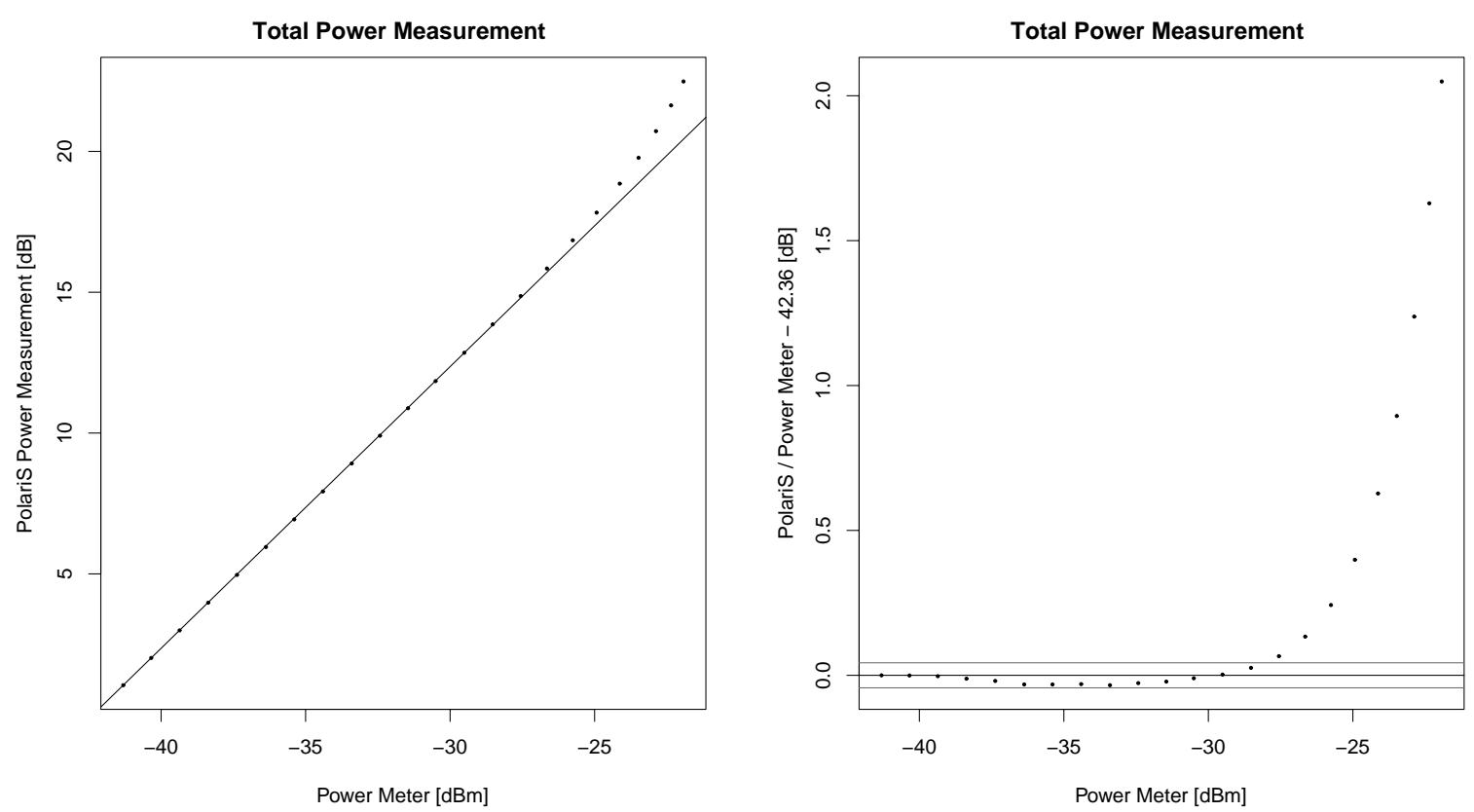

Fig. 6. (Left) Total power measurements by PolariS as a function of input power. The solid diagonal line indicate the best-fit linear relation with the slope of unity. (Right) Departure from the linear relation. The gray horizontal lines indicate departure of $\pm 1 \%$ range.

where $c$ is the sensitivity coefficient that corresponded to $42.46 \mathrm{~dB}$. Relative departure from the linear regression, $\frac{\log P_{\text {Polaris }}-\left(\log P_{\text {in }}+c\right)}{\log P_{\text {in }}+c}$, was within $1 \%$ in the range of $>13 \mathrm{~dB}$.

\subsection{Stability}

Time stability of the transfer function of the system is required for accurate measurements in radio astronomy observations that take a long integration time. Instability of a spectrometer can cause systematic errors when scans for calibrator and target sources are not simultaneous. In the case of radio spectroscopy, a spectrum acquired toward the blank sky (off-source) is used to calibrate the bandpass shape, $H(\nu)$, and to subtract the system noise (Yamaki et al., 2012). Stability of the bandpass shape is essential for weak-spectralline detectability.

To evaluate the bandpass stability, we measured the time-based Allan variance (TAV) and estimated the stable timescale. We employed the Smoothed Bandpass Calibration (SBC) method (Yamaki et al., 2012) to distinguish the systematic bandpass variation from random noise. The spectral-smoothing window was determined by the spectral flatness evaluated using the spectral Allan variance (SAV).
To evaluate the TAV and SAV, we conducted spectroscopy of the noise-source signals for $54,000 \mathrm{~s}$. The configuration is again shown in Fig. 3. Note that the measured instability involves not only PolariS but also the whole system.

\subsubsection{Time-based Allan variance}

For the evaluation of time stability, we calculated the $\mathrm{TAV}, \sigma_{\mathrm{y}}^{2}(\tau)$ at the reference spectral channel near the band center, as a function of time lag, $\tau$, defined as:

$$
\sigma_{\mathrm{y}}^{2}(\tau)=\frac{\left\langle\left[P_{\mathrm{ref}}(t+\tau)-2 P_{\mathrm{ref}}(t)+P_{\mathrm{ref}}(t-\tau)\right]^{2}\right\rangle}{2 \tau^{2}} .
$$

The TAV will follow $\sigma_{\mathrm{y}}^{2}(\tau) \propto \tau^{-2}$ while the systematic variation is not significant compared with the white random noise. We defined the timescale of stability when the excess of $\sigma_{\mathrm{y}}^{2}(\tau)$ from the $\propto \tau^{-2}$ relation is identical to the expectation.

Figure 7 shows the total power variation and the TAV measurement. The total power in the first $1000 \mathrm{~s}$ gradually increased. The variation can be ascribed to warming up of the noise source. The TAV followed the $\propto \tau^{-2}$ relation for $\tau \leq 256 \mathrm{~s}$ and the timescale of stability was $512 \mathrm{~s}$. Note that the TAV included not only variations of PolariS but also the analog devices such as the noise source, 

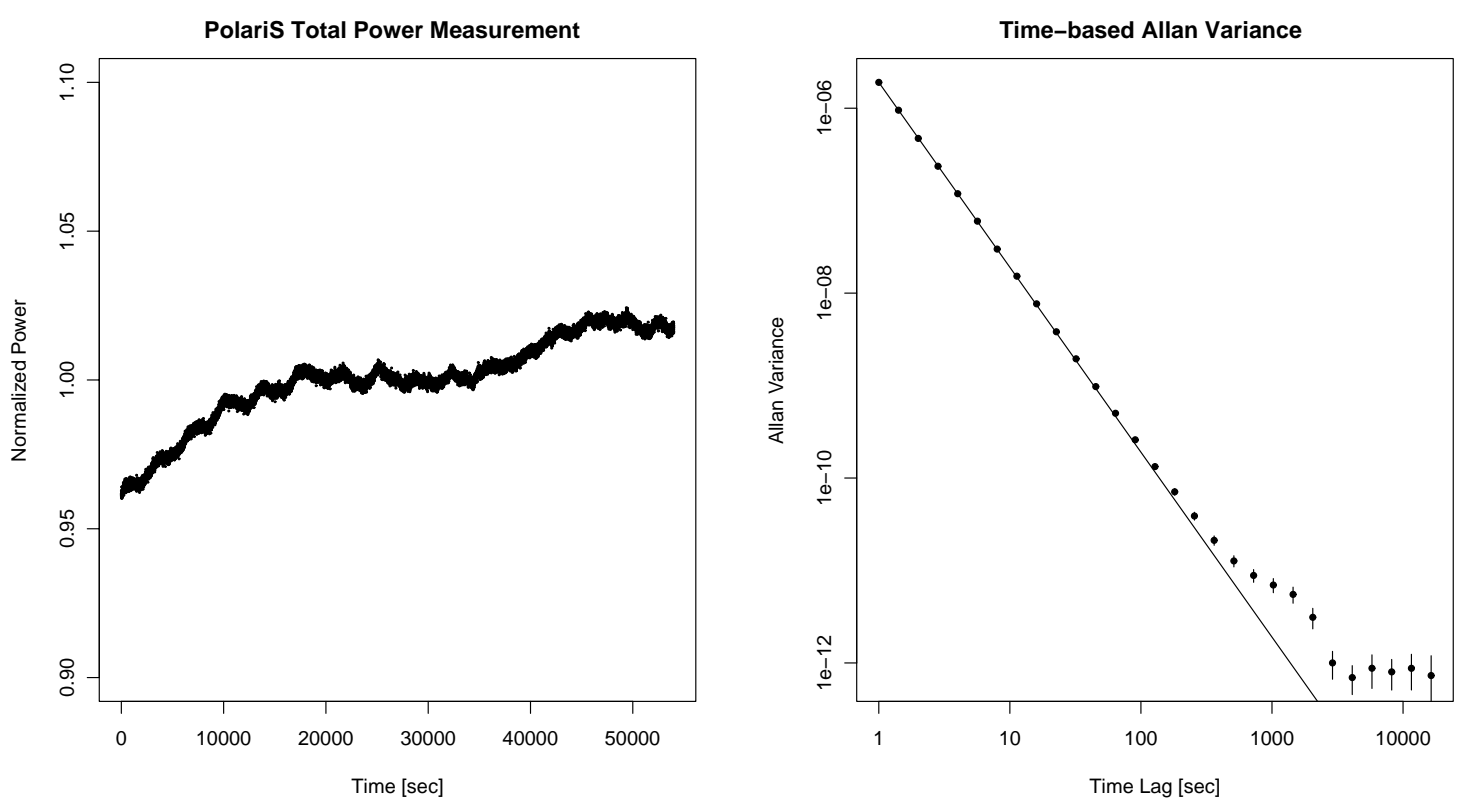

Fig. 7. (Left): Time variation of the total power measured by the histogram of digitized signals for $54,000 \mathrm{~s}$ with a 1 -s time resolution. The total power was scaled by the mean value. (Right): TAV of the total power measurement. The solid line indicates the power law with the power index of -2 , expected for white noise. The departure of TAV from the power law became significant at the time lag $\geq 512 \mathrm{~s}$.

the IF downconverter, and the baseband converter. The stability of solo PolariS must be better than the measured TAV.

\subsubsection{Bandpass stability}

The stability of bandpass shape was examined with the same configuration of the TAV, shown in Fig. 3. The overall bandpass table, $H(\nu)$ was obtained by integrating the spectrum for $54,000 \mathrm{~s}$ and then applying spline smoothing with a 128-ch width except for $6 \%$ band edges at both ends as shown the gray line in Fig. 9. Time series of 1800-s integrated bandpass variation, $\hat{H}_{k}(\nu)=\frac{H_{k}(\nu)}{G_{k} H(\nu)}$, were shown in Fig. 8 to evaluate the bandpass stability. Here, $k=1, \ldots, 30$ is the index of 1800 -s integration periods, and $G_{k}$ is the gain table obtained by 1800 -s integration of the total power, $P_{\text {Polaris }}$. To reduce random error in the bandpass variation, we averaged $\hat{H}_{k}(\nu)$ for 256 spectral channels $(15.625 \mathrm{kHz})$. The mean and SD values of each bandpass variation are attached in Fig. 8 and their time variations are plotted in Fig. 9. We did not find any significant systematic variation of the bandpass shape which exceeded the random-noise SD which was expected as $\frac{1}{\sqrt{\Delta \nu T}}=2.6 \times 10^{-4}$.

We found a decline of the mean value through the entire period in Fig. 9, and will discuss about it in Sec. 5.

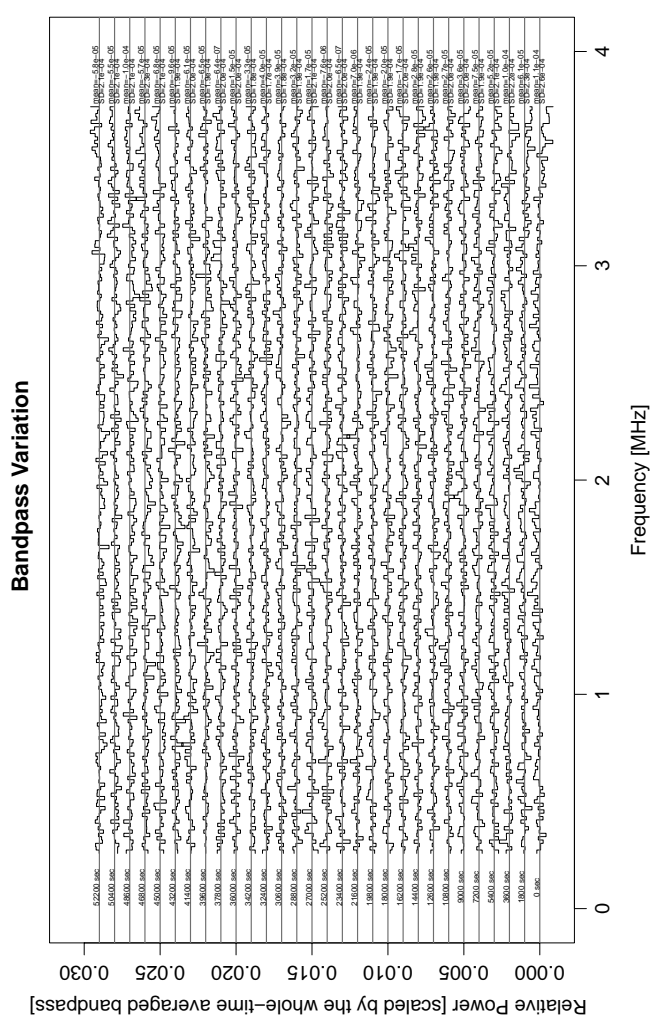

Fig. 8. Time variation of bandpass shape. The series of bandpass-corrected spectra, $\hat{H}_{k}(\nu)(k=1, \ldots, 30)$, are plotted with the offset baselines by the gray horizontal lines. The scale of the vertical axis is relative to the 54,000-s averaged bandpass, $H(\nu)$. Relative time from the start is labeled at the left of each spectrum, together with the mean and $\mathrm{SD}$ values at the right. 

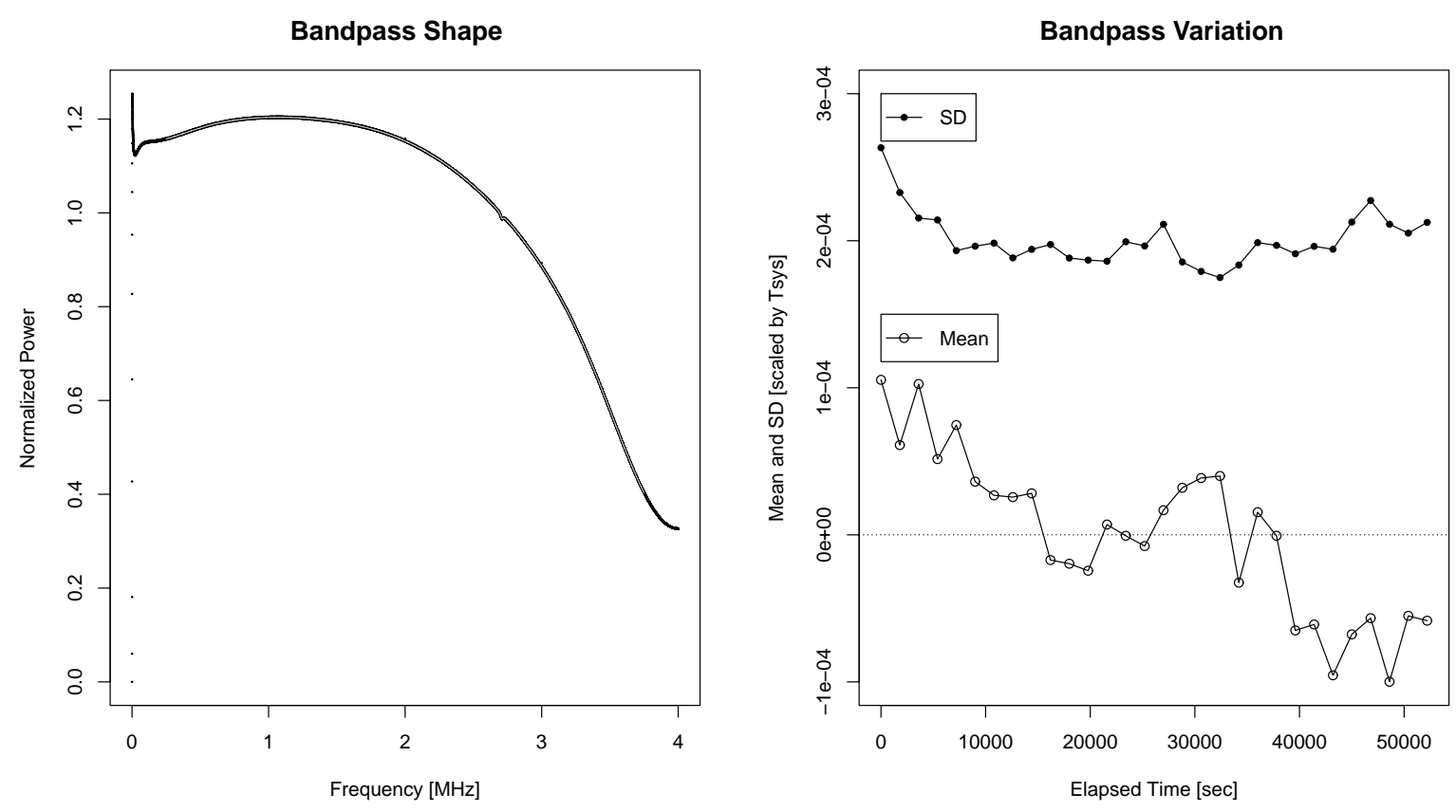

Fig. 9. (Left): Whole-bandwidth spectrum integrated for 54,000 s. The gray solid line indicates spline-smoothed bandpass shape without $6 \%$ band edges at both ends. (Right): Mean and SD values of calibrated spectra shown in Fig. 8 , as a function of relative time from the start.

\subsubsection{Spectral Allan variance}

The SAV defined by Eq. (4) is an indicator of bandpass flatness as a function of frequency separation, $\Delta \nu$.

$\sigma_{\mathrm{y}}^{2}(\Delta \nu)=\frac{\left\langle[H(\nu+\Delta \nu)-2 H(\nu)+H(\nu-\Delta \nu)]^{2}\right\rangle}{2 \Delta \nu^{2}}$.

While a spectrum is dominated by white noise, the SAV is given by the SD of bandpass-corrected spectrum, $\sigma=\sqrt{\left\langle H^{2}(\nu)\right\rangle-\langle H(\nu)\rangle^{2}}$, and the frequency separation as $\sigma_{\mathrm{y}}^{2}(\Delta \nu)=\frac{3 \sigma^{2}}{\Delta \nu^{2}}$ and it follows the power law of the frequency separation with the power index of -2 . When systematic distortion of the bandpass shape exceeds the white noise, on the other hand, the SAV shows a greater power index than -2 .

Figure 10 (right) shows the measurements of SAV, together with corresponding bandpass shapes in the left. The SAV with a short $(1 \mathrm{~s})$ integration was dominated by white noise that yields the power index of -2 in SAV. Time integration for 1800-s reduced the overall SAV, and it made the bandpass undulation apparent at $\Delta \nu \geq 4 \mathrm{ch}$ and became dominant at $\Delta \nu \geq$ $2048 \mathrm{ch}$. The systematic undulation component eliminated from the SAV after bandpass calibration was applied. The random noise component in the bandpass-calibrated spectrum was efficiently reduced by applying SBC.

\subsection{Sensitivity}

The sensitivity of a spectrometer is determined by the SD of line-free spectral channels after bandpass calibration and system-noise subtraction because detection of emission (or absorption) lines is judged by the signal-to-noise ratio (SNR), which is the ratio of line intensity to the SD. When the SD is dominated by random noise, the SD will be reduced by long time integration as $\sigma(T) \propto T^{-1 / 2}$, where $\sigma$ is the SD with respect to the system noise and $T$ is the integration time.

To evaluate the sensitivity, we used the same dataset for the SAV, bandpass stability, and TAV evaluations and measured the SD using PolariS for time integration up to $54,000 \mathrm{~s}$. The bandpass calibration table was generated from the spectrum integrated for $1800 \mathrm{~s}$ and applied the SBC. The SD of the spectrum was calculated in the frequency range of $1-2 \mathrm{MHz}$ where the bandpass shape was flat and no significant spurious signals were detected.

Figure 11 shows the results of the measurements. The SD at 1-s integration, scaled by the system noise, was 0.110 , which was $13.8 \%$ as small as the theoretical value of $\sigma=\frac{1}{\sqrt{\Delta \nu T}}$. At $54,000-\mathrm{s}$ integration, the SD decreased down to $4.82 \times 10^{-4}$ 

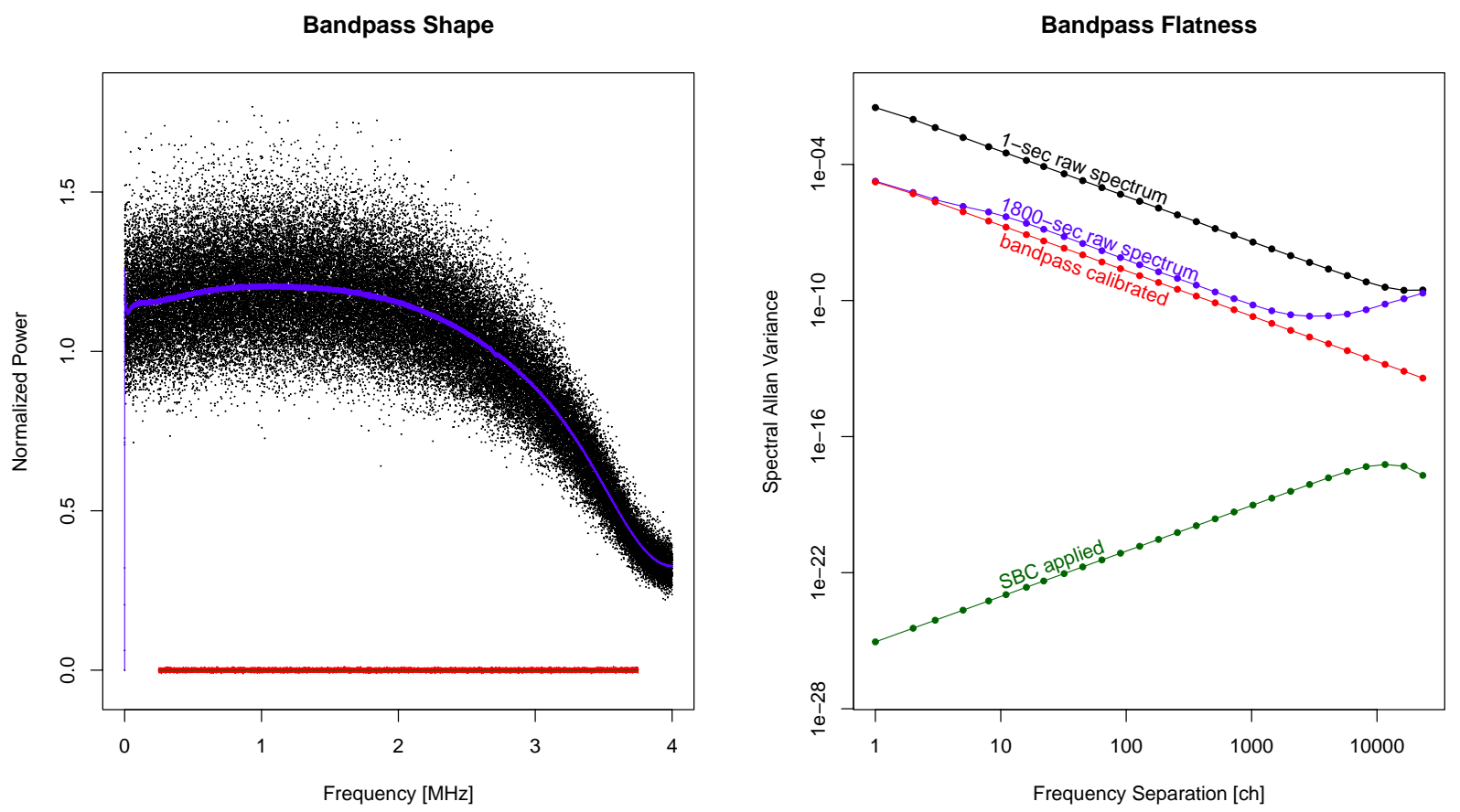

Fig. 10. (Left): Spectra at steps of bandpass calibrations. Black and blue dots indicate the raw spectra before and after 1800$\mathrm{s}$ integration, respectively. The red dots indicate the bandpass-calibrated spectrum. The SBC-applied spectrum is plotted in the green line. (Right): SAVs. Color indices are the same situation with the spectra in the left panel.
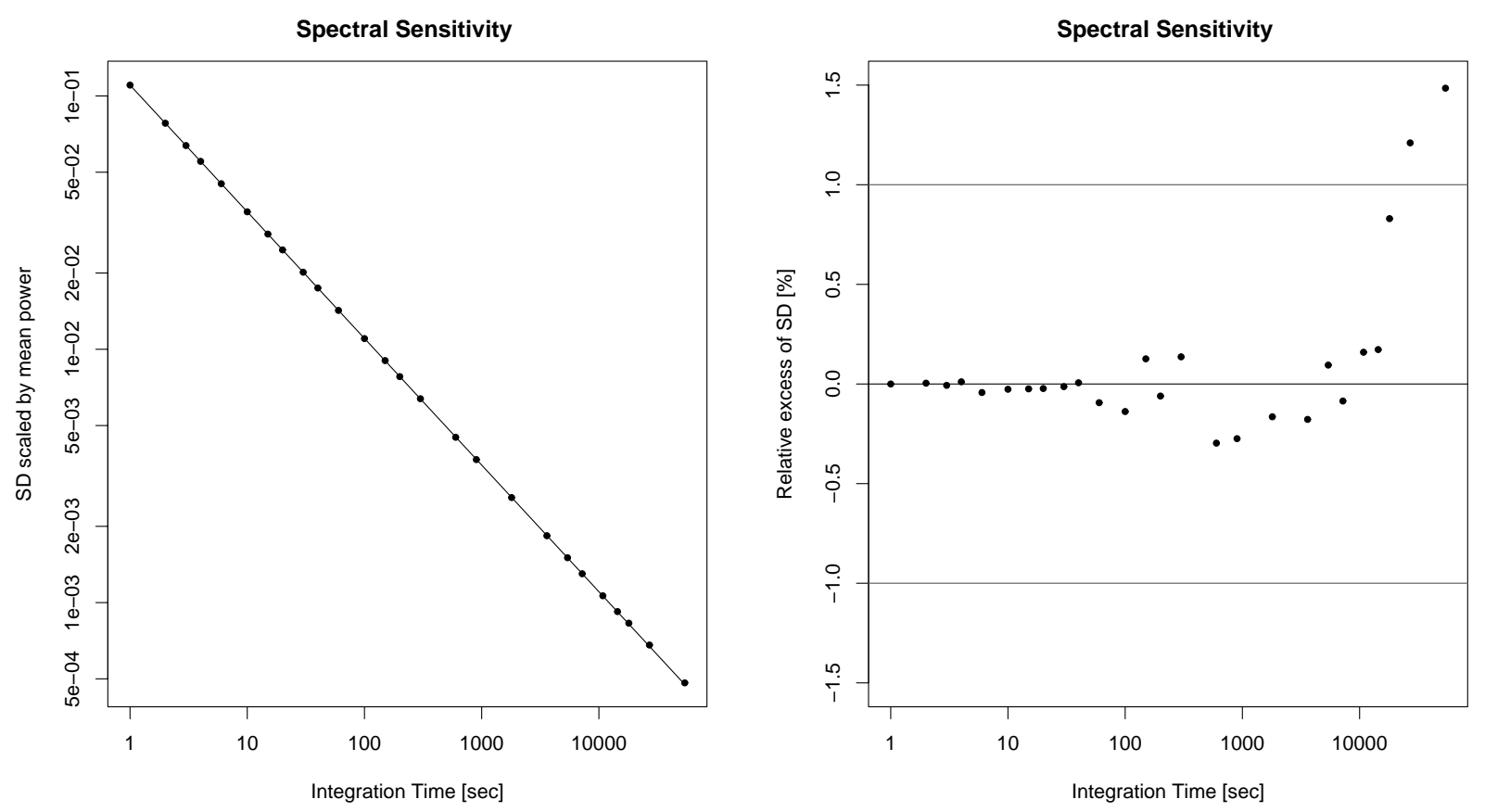

Fig. 11. (Left): The root-mean-square (RMS) of bandpass-corrected spectra in the frequency range of $1-2 \mathrm{MHz}$ with various integration time, $T$, ranged $1-54,000 \mathrm{~s}$. The solid diagonal line indicates extrapolation from the RMS at $1 \mathrm{~s}$ with the $\propto 1 / \sqrt{T}$ law. (Right): Relative excess of the RMS from the $\propto 1 / \sqrt{T}$ law. The gray horizontal lines indicate $\pm 1 \%$ range from the expectation. 

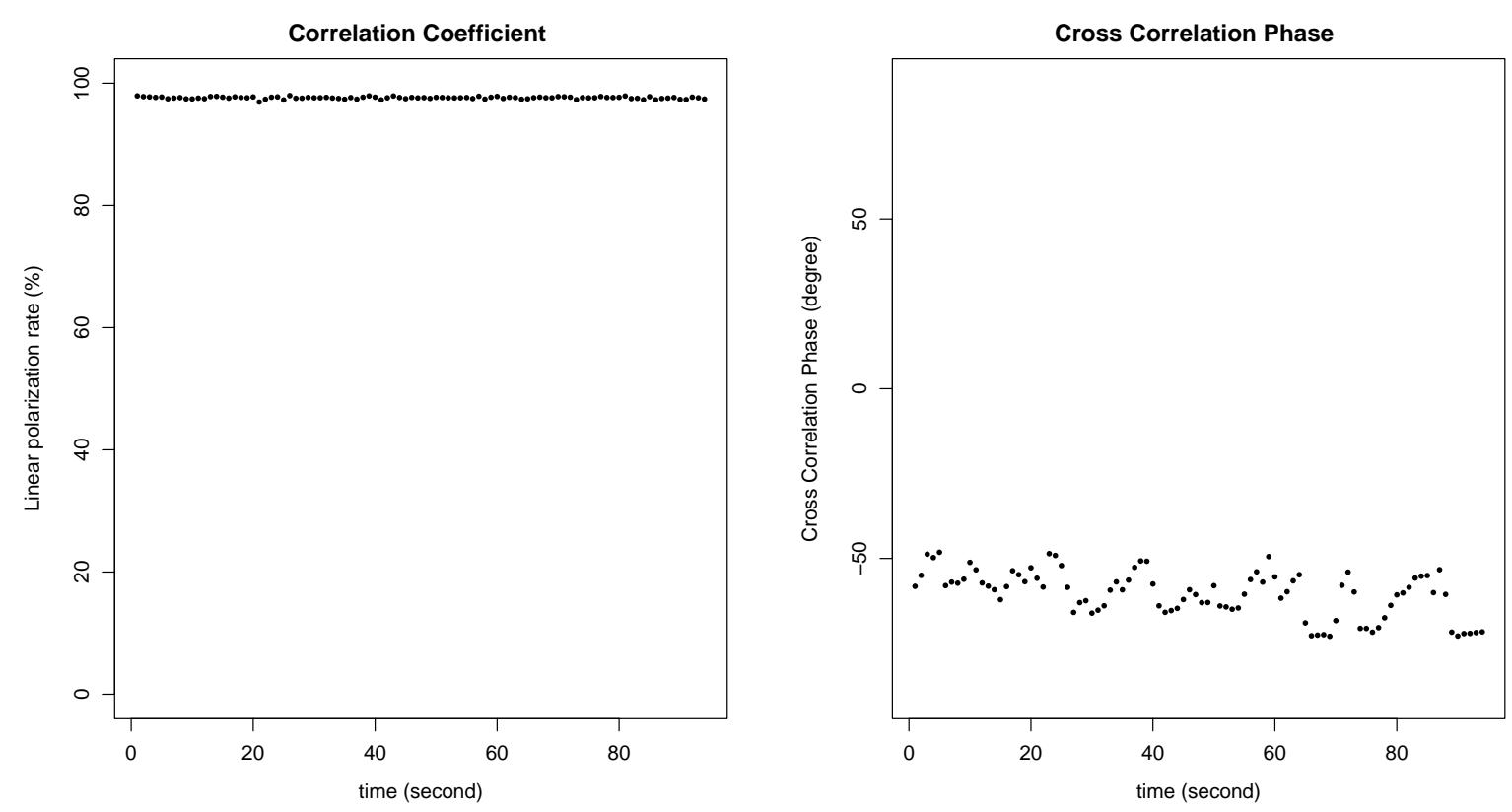

Fig. 12. (Left): Correlation coefficient between two circular polarization signals taken by PolariS. The input signal was the linearly polarized monochromatic wave, which was expected to perfectly correlate. (Right): Phases of the correlation function, which corresponds to relative phase between two circular polarization signals. The SD of the phase was $-60^{\circ} .4 \pm 6^{\circ} .8$.

which was still $12.5 \%$ as small as the theoretical expectation. If we use the SD at $1 \mathrm{~s}$ as the reference (see the right of Fig. 11), the SD follows the $\propto T^{-1 / 2}$ law up to the integration of $18,000 \mathrm{~s}$. within $1 \%$ excess.

\subsection{Cross-correlation}

The cross-correlation function between two orthogonal polarizations is necessary to obtain full Stokes spectra, as stated in Sec. 2.1. To verify the cross products of PolariS, we used the H22 dual circular polarization receiver and injected a linearly polarized monochromatic wave at $22.233 \mathrm{GHz}$, generated with a signal generator (Agilent 83650L) and a transducer with a rectangular waveguide. The received two circular polarization signals which must correlate each other were transmitted to the VLBI backend, and acquired with PolariS. The whole configuration is shown in Fig. 3, too. The cross-correlation products of PolariS were tested in terms of the correlation coefficient and relative phase for $94 \mathrm{~s}$ with the time resolution of $1 \mathrm{~s}$. The results are shown in Fig. 12. The correlation coefficient was $97.6 \pm 0.2 \%$ and the SD of phase was $6.8^{\circ}$ during the observation of $94 \mathrm{~s}$. We discuss the reason why the correlation differs from $100 \%$ in Sec. 5.

\section{Field Test Observations}

For the purpose of commissioning and science verification, we tried test observations of $\mathrm{SiO}$ maser lines toward the Mira variable star, R Leo, and the CCS molecular line toward the star-forming molecular core in the TMC-1 using the $\mathrm{Z} 45$ receiver on the Nobeyama 45-m radio telescope.

For $\mathrm{R}$ Leo, two transitions of $\mathrm{SiO} J=1-0$, $v=1(43.122 \mathrm{GHz})$ and $v=2(42.821 \mathrm{GHz})$ were simultaneously observed with the dual linear polarizations on 2014 March 16. Spectroscopy was originally carried out with 16,384 channels across $4-\mathrm{MHz}$ bandwidth (244-Hz resolution) and channelaveraged into $3.9-\mathrm{kHz}$ resolution. The integration time for on- and off-source were 41 and $65 \mathrm{~s}$, respectively, and the off-source spectra were subtracted from the on-source ones. The power spectra and cross power spectra are shown in Fig. 13. The power spectra of $\left\langle X X^{*}\right\rangle$ and $\left\langle Y Y^{*}\right\rangle$ were significantly different and the cross power spectra were significantly detected.

For the TMC-1, the CCS $J_{N}=4_{3}-3_{2}$ transition at $45.379 \mathrm{GHz}$ was observed on 2013 December 23. We pointed to (RA, DEC) $)_{22000}=$ $\left(04^{h} 41^{m} 42^{s} .47,+25^{\circ} 41^{\prime} 27^{\prime \prime} .1\right)$, which was close to 'b7' clump of TMC-1 core D region (Peng et al., 1998). Spectroscopy with integration of 252 and $375 \mathrm{~s}$ for on- and off-source was carried out with 

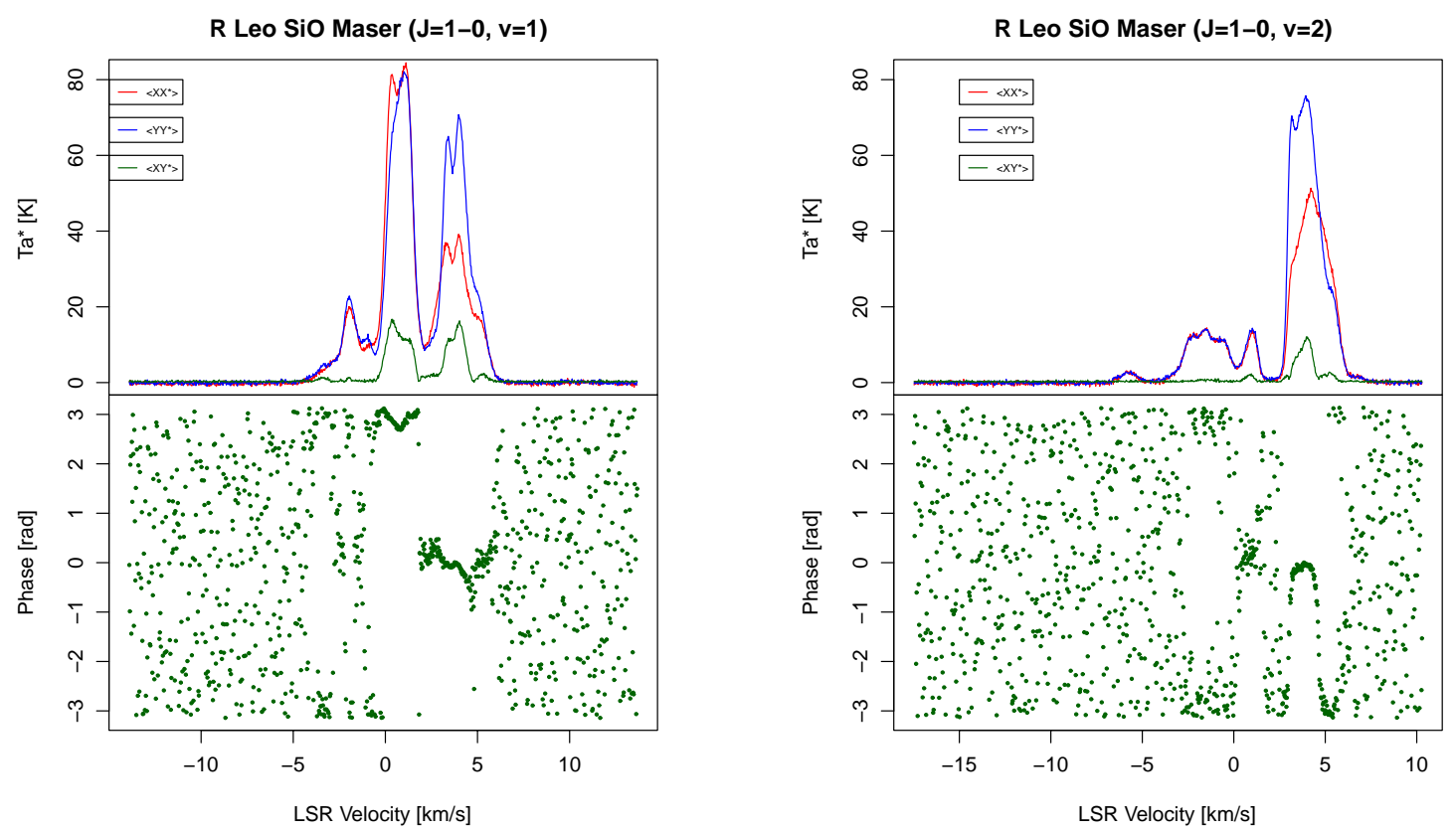

Fig. 13. Power spectra and cross power spectra of $\mathrm{SiO}$ maser $(J=1-0)$ emission lines toward R Leo. Left and right panels indicate the vibration state of $v=1$ and $v=2$ at 43.122 and $42.821 \mathrm{GHz}$, respectively. Red and blue solid lines in the top stand for the power spectra of $X$ and $Y$ linear feeds, respectively. Green solid line and filled circles indicate the amplitude and phase of the cross power spectrum between $X$ and $Y$ polarizations.

131,072 channels across 8-MHz bandwidth $(61-\mathrm{Hz}$ resolution) and channel-averaged into $1.95-\mathrm{kHz}$ resolution. Since a relatively strong spurious signal appeared in one spectral channel near the center of CCS line profile, we flagged the channel before

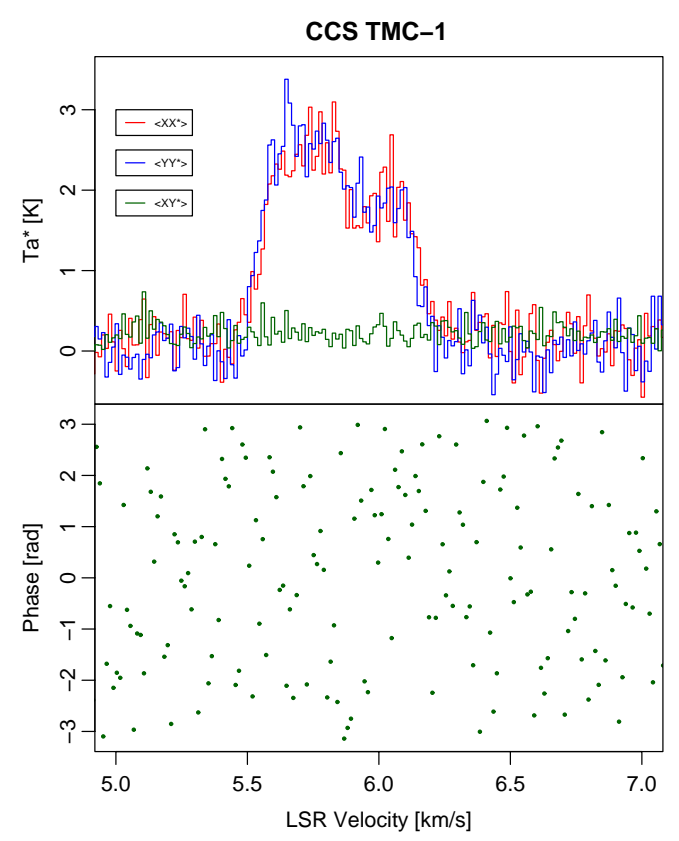

Fig. 14. Power spectra and cross power spectra of CCS emission line toward TMC-1 at the spectral resolution of $1.95 \mathrm{kHz}$. Color assignments are the same with Fig. 13. spectral averaging. The power spectra and cross power spectra are shown in Fig. 14.

\section{Discussion}

The engineering tests of PolariS presented here demonstrate compliance with specifications.

The spectral resolution perfectly coincided with the theoretical expectation, as shown in Sec. 3.1. The current software employs the boxcar window function in the time-domain FFT segments, which results in the sinc-squared spectral resolution function. This treatment causes subtle sidelobe associated with narrow and strong emission lines. PolariS is a software spectrometer, which is flexible for applying other window functions, such as Hamming, Hanning, and Blackman windows, to reduce sidelobe levels in the spectral resolution function. We choose the boxcar window for online processing because sidelobe mitigation with a relevant window function can be applied in post processing. Observers can apply desired apodization function or spectral smoothing to reduce spectral sidelobes.

The system marked sufficient linearity in total power measurements within $1 \%$ accuracy over the input power range of $13 \mathrm{~dB}$. The total power measurement is used to estimate the receiver noise 
temperature and the system noise temperature by comparing powers in two or more circumstances where the feed horn points to the absorber at ambient temperature $(\sim 293 \mathrm{~K})$, to the cold load $(\sim 77 \mathrm{~K})$, and to the blank sky $(\sim 20 \mathrm{~K})$. Since the typical receiver noise of the $\mathrm{Z} 45$ is $\sim 50 \mathrm{~K}$, the power range between the cold load, the blank sky, and the ambient load will be $\frac{293+50}{77+50}=2.7$ $(4.3 \mathrm{~dB})$ and $\frac{293+50}{20+50}=4.9(6.9 \mathrm{~dB})$. Thus, the dynamic range of $>13 \mathrm{~dB}$ is sufficient for the system noise measurement, if we set the power level adequately.

We note that the departure from linearity displayed in Fig. 6 could be due to saturation of the power meter, because the power meter we employed for this experiment has a maximum rated input power of $-20 \mathrm{dBm}$. Therefore, the linear range of PolariS could be wider than presented here.

The dynamic range of $33 \mathrm{~dB}$ in line intensity is also enough for astronomical spectroscopy. The departure from the linear trend in high-power ends can be ascribed to inaccuracy of the signal generator, because the sensitivity profiles above $-40 \mathrm{dBm}$ are very similar in both "high-power" and "low-power". The underestimation in low-power ends, especially in the "low-power" ATT setting, can be caused by uncertainty in baseline subtraction because the departure from the linear trend was significant where the line intensity is comparable to the $\mathrm{SD}\left(=\frac{1}{\sqrt{\Delta \nu T}}\right)$ of the system noise.

The performance of weak line detectability is verified with the SD of the spectrum at long integration time. As is shown in Sec. 3.4, the sensitivity is maintained for the timescale of $54,000 \mathrm{~s}$. The field-test observations of CCS molecular line toward TMC-1 also indicate that the system shows no significant departure in the linearity range down to $T_{\mathrm{a}}^{*} / T_{\mathrm{sys}}=1.7 \%$.

As shown in Sec. 3.3, the bandpass shape is very smooth and stable, allowing us to apply SBC to reduce random errors in off-source scans efficiently. This indicates that the analog system (baseband converters and ADC) performs sufficiently stably and the digital system (in software) works perfectly. The SAV (see Fig. 10) indicates a very good flatness in the bandpass-corrected spectrum. The systematic error of bandpass shape after SBC application will be less than $\sqrt{\frac{2 \Delta \nu^{2} \mathrm{SAV}}{3}}=2.3 \times 10^{-5}$ at the maximum channel separation of $11,585 \mathrm{ch}$ for broad line detection, even if we employ a single bandpass table for 15-hr observations.
The mean values of the bandpass-corrected spectra were decreasing while increasing the total power (see Fig. 7). We consider that this is ascribed to increase of power in band edges caused by bandpass distortion. Because the gain table, $G(t)$, is derived from bit distribution that includes power in band edges, increase of power in band edges makes $G(t)$ greater and lets the mean power without band edges decreased after gain calibration. We can also see convex and concave distortion at the beginning and ending periods, respectively, in Fig. 8. This behavior supports the explanation above.

The stability in bandpass shape is also confirmed by the sensitivity shown in Sec. 3.4. As shown in Fig. 11, the SD of bandpass-corrected spectra follows the $\propto T^{-1 / 2}$ law for the integration time shorter than $18,000 \mathrm{~s}$. The excess of SD at the longest integration time can be caused by the bandpass error, related with variation in total power as discussed in the last paragraph, because the distortion of bandpass shape after SBC is estimated to $\sim 10^{-5}$ and non-negligible for integration longer than $18,000 \mathrm{~s}$. Note that the SD value at the longest integration time is still smaller than the expectation of $\frac{1}{\sqrt{\Delta \nu T}}$. This is probably because the effective bandwidth is narrower than $4 \mathrm{MHz}$ due to the antialias filter (see Fig. 10) and thus the sampling frequency of $8 \mathrm{MHz}$ is slightly higher than the Nyquist rate.

The cross-correlation capability is confirmed by the engineering test with the artificial source (Sec. 3.5) and the field test (Sec. 4). With the injection of the linearly polarized artificial monochromatic wave into a dual circular feed, we got $97.6 \pm 0.2 \%$ of correlation. The departure from $100 \%$ could be due to temperature fluctuation of the system that yields relative phase fluctuation between LHCP and RHCP.

The measured cross power spectra of the $\mathrm{SiO}$ maser in the observation toward R Leo (see Fig. 13) demonstrates the potential of this instrument to perform high precision polarimetric observations. In both $v=1$ and $v=2$ transitions, the velocity components within $0 \leq V_{\mathrm{LSR}} \leq 7 \mathrm{~km} \mathrm{~s}^{-1}$ shows highly polarized emission with a significant amplitude in cross power spectra, small phase dispersion, and difference between $\left\langle X X^{*}\right\rangle$ and $\left\langle Y Y^{*}\right\rangle$ spectra. The large gap in phase between velocity components of $0 \leq V_{\mathrm{LSR}} \leq 2 \mathrm{~km} \mathrm{~s}^{-1}$ and $2 \leq V_{\mathrm{LSR}} \leq 7 \mathrm{~km} \mathrm{~s}^{-1}$ in $v=1$ suggests that the electric-vector position angles are significantly different between these two 
components. The blueshifted components in $-7 \leq$ $V_{\mathrm{LSR}} \leq 0 \mathrm{~km} \mathrm{~s}^{-1}$ show much weaker polarization than the redshifted components.

The CCS molecular emission is expected to be unpolarized, because it is a thermal emission in the dark cloud. As was expected, we got neither significant detection in cross power spectrum nor significant difference between $\left\langle X X^{*}\right\rangle$ and $\left\langle Y Y^{*}\right\rangle$ spectra (see Fig. 14). The phases of the cross power spectra randomly distributed in $[-\pi, \pi]$, suggesting nondetection. Note that the amplitude of cross power spectrum is biased from 0 , because it is always positive and follows the Rayleigh distribution when the expectation of cross power spectrum is 0 .

The line peak intensity of $T_{\mathrm{a}}^{*}=2.57 \pm 0.04 \mathrm{~K}$ with $1.95-\mathrm{kHz}$ resolution is slightly higher than the results of $T_{\mathrm{a}}^{*}=2.23 \pm 0.09 \mathrm{~K}$ with $37-\mathrm{kHz}$ resolution using the acousto-optical spectrometer (AOS) on the Nobeyama 45-m radio telescope (Suzuki et al., 1992). If we apply channel-averaging to set the same spectral resolution of $37 \mathrm{kHz}$, the spectrum by PolariS results in $T_{\mathrm{a}}^{*}=2.47 \pm 0.04 \mathrm{~K}$ and close to the AOS measurement. The line profile from the mapping observation of TMC-1 (Peng et al., 1998) showed a somewhat different profile, depending on the position of the molecular cloud. Because of the difference of beam size and pointing position, it is difficult to evaluate the difference of line profiles.

The widths of both line edges are only $\sim 20 \mathrm{~Hz}$. The sharpness of the line profile was $\frac{d I}{d \nu}=$ $0.16 \mathrm{mK} \mathrm{Hz}^{-1}$ that yields Stokes $V \pm 10 \mathrm{mK}$ at both line edges if we assume the Zeeman shift of $64 \mathrm{~Hz}$ for $B \sim 100 \mu \mathrm{G}$. To detect the Stokes $V$ with the SNR of 5 under the condition of $T_{\mathrm{sys}}=100 \mathrm{~K}$, on-source integration time of $\left(5 \times \frac{100 \mathrm{~K}}{10 \mathrm{mK}}\right)^{2} \frac{1}{2 \times 20 \mathrm{kHz}}=62,500 \mathrm{~s}$ is required.

\section{Summary}

We have developed a software-based polarization spectrometer, PolariS, using commercially available devices of the K5/VSSP32 sampler and the Linux computer, at the cost of JPY 500,000 and JPY 70,000, respectively. Using the GPU for FFT and cross-correlation processes, spectroscopy for 4-IF 4or $8-\mathrm{MHz}$ bandwidth with a $61-\mathrm{Hz}$ spectral resolution can be carried out in real time.

Through the engineering tests, we have verified the basic performance of PolariS. The spectral resolution function was consistent with the theoretical prediction of a sinc-squared profile with a
FWHM of $54.02 \pm 0.06 \mathrm{~Hz}$. The linearity between input signal power and measured power has been confirmed within $1 \%$ accuracy for the range of $>13 \mathrm{~dB}$ and $33 \mathrm{~dB}$ for continuum total power and emission line, respectively. The bandpass shape was flat and stable enough to apply SBC with $125-\mathrm{kHz}$ frequency width. No significant bandpass variation exceeding $2.6 \times 10^{-4}$ with respect to the system noise was found for the time span of $15 \mathrm{~h}$. The SD of the spectrum was $12.5 \%$ as small as the expectation of $\frac{1}{\sqrt{\Delta \nu T}}$ at 54,000-s integration and follows the $\propto T^{-1 / 2}$ law between 1 and $54,000 \mathrm{~s}$. The cross power spectra were verified by injection of a linearly polarized artificial signal into a dual circular feed and cross-correlation of them.

The field test observations were carried out toward the strongly polarized maser source, R Leo, and unpolarized CCS emission of TMC-1. We verified the performance of the power spectra that will yield full Stokes spectra crucial to our primary aim of the Zeeman effect measurements.

All the software code is open in the GitHub repository and anyone can download and use it or review the signal processing inside.

\section{Acknowledgments}

The development of Polaris is supported by the FY2012 Joint Development Research program of the National Astronomical Observatory of Japan, and by a Grant-in-Aid for Scientific Research of Japan (24244017). We thank the staff and students in the Department of Physics and Astronomy, Kagoshima University for assistance with fundamental tests using the VERA Iriki station. We are grateful to the staff of the Mizusawa VLBI Observatory for technical support and commissioning. The authors thank the staff at the Nobeyama Radio Observatory (NRO) for operations of the 45 -m radio telescope in the commissioning science verification. NRO is a division of the National Astronomical Observatory, National Institutes of Natural Sciences, Japan.

\section{References}

Baart, E. E., Cohen, R. J., Davies, R. D., Rowland, P. R. \& Norris, R. P. [1986] MNRAS 219, 145.

Barsdell, B. R., Bailes, M., Barnes, D. G. \& Fluke, C. J. [2012] MNRAS 422, 379.

Crutcher, R. M., Troland, T. H., Goodman, A. A. et al. [1993] ApJ 407, 175 . 
Crutcher, R. M., Troland, T. H., Lazareff, B., Paubert, G. \& Kazès, I. [1999] ApJL 514, L121.

Dowd, A., Sisk, W. \& Hagen, J. [2000] "WAPP — Wideband Arecibo Pulsar Processor," in Proc. IAU Colloq. 177, Pulsar Astronomy - 2000 and Beyond, eds. Kramer, M., Wex, N. \& Wielebinski, N., San Francisco, ASP Conf. Ser., Vol. 202, p. 275.

Fiebig, D. \& Guesten, R. [1989] A\&A 214, 333.

Gaume, R. A. \& Mutel, R. L. [1987] ApJS 65, 193.

Goodman, A. A., Crutcher, R. M., Heiles, C., Myers, P. C. \& Troland, T. H. [1989] ApJL 338, L61.

Kondo, T., Koyama, Y., Takeuchi, H. \& Ichikawa, R. [2006a] IVS NICT-TDC News 27, 5.

Kondo, T., Koyama, Y., Takeuchi, H. \& Kimura, M. [2006b] "Development of a new VLBI sampler unit (K5/VSSP32) equipped with a USB 2.0 interface," in IVS 2006 General Meeting Proc., Concepción, Chile, p. 195.

Lo, K. Y., Walker, R. C., Burke, B. F. et al. [1975] ApJ 202, 650.

Magro, A., Karastergiou, A., Salvini, S. et al. [2011] MNRAS 417, 2642.

Nakamura, F., Ogawa, H., Kameno, S. et al. [2013] ASP Conf. Ser. 476, 239.

Nakatake, A., Kameno, S. \& Takeda, K. [2010] PASJ 62, 1361.

Norris, R. P. [1984] MNRAS 207, 127.
NVIDIA Corporation [2007] CUDA Parallel Programming and Computing Platform, http://www.nvidia.com/ object/cuda_home_new.html.

Onishi, T., Mizuno, A., Kawamura, A., Tachihara, K. \& Fukui, Y. [2002] ApJ 575, 950.

Peng, R., Langer, W. D., Velusamy, T., Kuiper, T. B. H. \& Levin, S. [1998] ApJ 497, 842.

Reid, M. J., Haschick, A. D., Burke, B. F. et al. [1980] ApJ 239, 89.

Shinnaga, H. \& Yamamoto, S. [2000] ApJ 544, 330.

Shu, F. H., Adams, F. C. \& Lizano, S. [1987] ARAA 25, 23.

Spitzer, L. [1968] Diffuse Matter in Space (Interscience Publication, New York).

Suzuki, H., Yamamoto, S., Ohishi, M. et al. [1992] ApJ 392, 551.

Thompson, A. R., Moran, J. M. \& Swenson, G. W. Jr. [2001] Interferometry and Synthesis in Radio Astronomy, 2nd edition (Wiley, New York).

Thum, C., Wiesemeyer, H., Paubert, G., Navarro, S. \& Morris, D. [2008] PASP 120, 777.

Tokuda, K., Kozu, M., Kimura, K. et al. [2013] ASP Conf. Ser. 476, 403.

Troland, T. H. \& Heiles, C. [1986] ApJ 301, 339.

Yamaki, H., Kameno, S., Beppu, H., Mizuno, I. \& Imai, H. [2012] PASJ 64, 118. 